\title{
A QoS-Aware Scheduling Algorithm Based on Service Type for LTE Downlink
}

\author{
Weihong Fu, Qingliang Kong, Weixin Tian, Cheng Wang, Lili Ma \\ State Key Lab. Of Integrated Service Networks, Xidian University \\ Xi'an, China \\ e-mail:whfu@mail.xidian.edu.cn
}

\begin{abstract}
In this paper, a resource scheduling algorithm for Long Term Evolution (LTE) downlink is proposed. It provides Quality of Service (QoS) guarantee to Guaranteed Bit Rate (GBR) services and also suit to the Non Guaranteed Bit Rate (Non-GBR). Different services possess different QoS needs in LTE system, based on this, 3GPP divide them into several types. In view of the division, we set priorities for different kinds of services to guarantee the transportation of GBR services which have high QoS needs. At the same time, we take the Media Access Control (MAC) layer logical channel priority into account, and consider users' channel quality to acquire a higher cell throughput. Simulation results show that our scheme can improve the QoS of emergency services obviously at the cost of a certain amount of the non-real times'.
\end{abstract}

Keywords-resource scheduling; GBR; Non-GBR; QoS; LTE

\section{INTRODUCTION}

In recent years, there appear some kinds of communication systems using Orthogonal Frequency Division Multiplexing (OFDM), for example, WiMAX and 3GPP LTE. They support different services and can achieve high throughput. On the other hand, these systems are able to improve cell throughput further by means of combing with techniques such as Multiple Input Multiple Output (MIMO) and Hybrid Automatic Repeat reQuest (HARQ). However, introduction of these new techniques makes higher requirement to the resource scheduling algorithms in MAC layer.

Traditional scheduling algorithms, including Maximum Carrier to Interference $(\max C / I)$, Round Robin $(R R)$ and Proportional Fairness (PF) [1][2], and its improved algorithms [3][4] have a common drawback. Non of them take QoS needs of user's services into consideration. Sometimes there are many users in the system and Non-GBR services need too much resource, in this case, this kind of algorithm can't guarantee the performance of GBR services.

There exist some algorithms considering user QoS for Orthogonal Frequency Division Multiple Access (OFDMA) system. One is Earliest Deadline First (EDF) [5], user with maximum packet delay has the highest priority, and this algorithm does best in packet delay and loss rate. Whereas, it can neither guarantee system throughput nor suit to non-realtime services. Another is Modified Largest Weighted Delay First (M-LWDF) [6] and its modified algorithms [7] [8]. This kind of algorithm adds packet delay to $\mathrm{PF}$, and combines it with channel quality information. Nonetheless, they neither consist of difference between logical channel prioritization nor apply to non-real-time service.

Therefore according to limitation of the above methods, we propose a novel scheduling algorithm. It combines service QoS and MAC logical channel prioritization with user channel quality indication and fairness between users. It applies to not only GBR bearers but also Non-GBR bearers.

The paper is organized as follows. In Section II, it provides the system model of resource scheduling in LTE system. Section III describes the proposed resource allocation algorithm. Section IV presents the simulation results and performance analysis. Finally Section V concludes this paper.

\section{SYSTEM MODEL}

Packet scheduling is to manage and schedule services according to their characteristics. When there are several packet streams waiting to be served, we should determine reasonable service rules to arrange the service order, service time and transmission rate to satisfy their QoS needs, this is the basic issue of packet scheduling. In LTE system, scheduler is the functional entity to achieve packet scheduling. To match different wireless channel data rate quickly, and meet the needs of different kinds of high speed packet service, it has been moved from Radio Network Controller (RNC) side in WCDMA to evolved NodeB (eNode B) side in LTE [11].

\section{A. Scheduler in LTE}

Scheduler is the functional entity to achieve fast packet scheduling and the core function of MAC layer in LTE eNode B [10]. It has a major impact on the performance of the system. It contains uplink scheduler and downlink scheduler, which take charge of allocating physical layer resources for Uplink Shared Channel (UL-SCH) and Downlink Shared Channel (DL-SCH) respectively. Physical layer resources mainly consist of Physical Resource Block (PRB), Modulation and Coding Scheme (MCS), power allocation schemes and antenna selection schemes in the multiple antenna case [9]. Fig. 1 shows the position of scheduler in LTE protocol and its relationship with key techniques like HARQ and Adaptive Modulation and Coding 
(AMC). Its key function is to determine how to allocate resource to users properly for the purpose of maximizing system throughput, on condition that ensure packet delay and QoS.

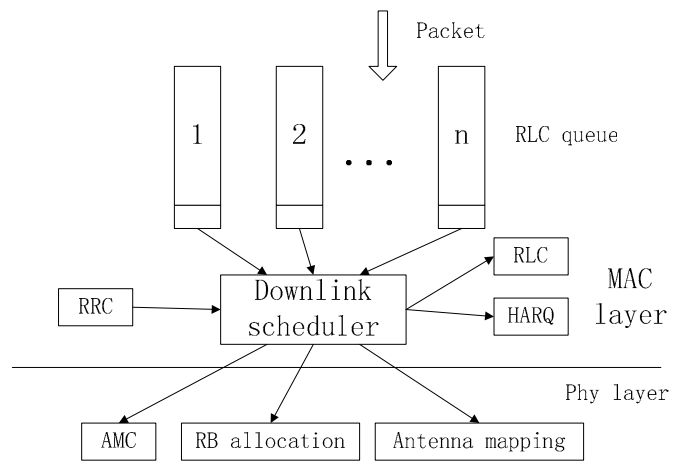

Figure 1. Position of scheduler in LTE protocol

\section{B. Downlink Scheduling Process in LTE}

As mentioned, channel quality is necessary in scheduling. eNode B obtains it by receiving feedback of channel quality indicator from user. As shown in Fig. 2, downlink scheduler allocates resource for users according to their channel quality, QoS needs and fairness. eNode B sends date and scheduling signaling according to the scheduling results. Scheduled users accept data in the light of RBs allocation results, MCS and antenna select options in downlink scheduling signaling.

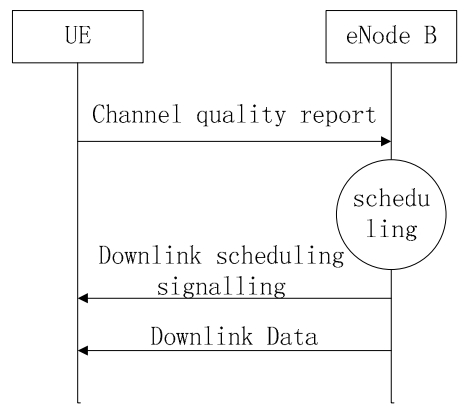

Figure 2. Downlink scheduling process

\section{DESCRIPTION OF RESOURCE SCHEDULING ALGORITHM}

The resource allocating algorithm that we will descript accomplishes the scheduling function in figure2. In the proposed algorithm, GBR service is definitely prior to NonGBR service, and resource allocation for Non-GBR service is behind of GBR.

Occasionally, there may be many users in the system and these who have terrible channel quality occupy too much resource to transport GBR data, thus lead to the reduction of system throughput. To prevent the appearance of this situation, we set a threshold $\alpha$, called percentage of the resource that GBR services can utilize. It signifies the permission of GBR services. When $\alpha$ is large, it means that there will be more resource that GBR services can use, and their QoS needs is able to be satisfied sufficiently; while $\alpha$ is small, the situation is reversed. $\alpha$ is an experience value, and is changeable. It can be set based on the number of users and percentage of GBR services in the system.

In the situation that there are no GBR resource while there still exist GBR services, we stop serving for them. If all of GBR services have been served before GBR resource is exhausted, the rest belong to the non-GBR.

The whole technological process is illustrated in Fig.3.

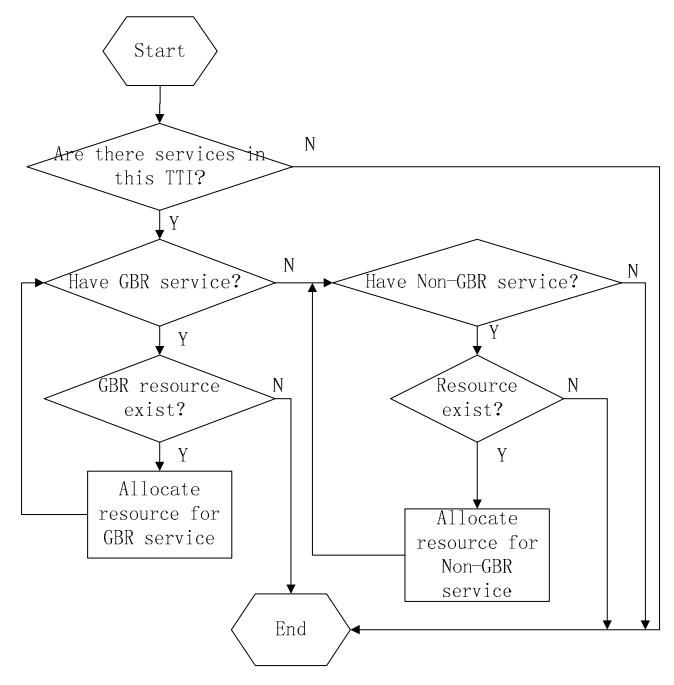

Figure 3. Block diagram of the algorithm

We will introduce our resource scheduling algorithm in two steps.

\section{A. Resource Allocation of GBR Services}

As before, if there are GBR services, we allocate resource for them preferentially. If all of the GBR available resource is exhausted, then serve Non-GBR services in turn.

When allocating resource for GBR services, we should take into account users' channel quality, packet delay and logical channel prioritization. For GBR services, we set the priority calculation formula as follows.

$$
F_{U}^{\mathrm{i}}=w_{i} * \frac{\left(R_{i} * E_{U}\right)}{H_{U}^{i}} * \frac{\mathrm{D}_{\mathrm{U}}^{\mathrm{i}}}{\mathrm{T}_{\mathrm{i}}}
$$

Where, $w_{i}$ is the weight factor of logical channel $i . R_{i}$ is the guaranteed bit rate of service $i . E_{U}$ is spectrum efficiency of user $U . H_{U}^{i}$ is the history throughput of 
service $i$, and $D_{U}^{i}$ is its queue delay. $T_{i}$ is the maximum delay that service $i$ can tolerant. We will explain the key factors as follows in detail.

Computing methods of $E_{U}$ and $H_{U}^{i}$ are the same as conventional $\mathrm{PF}$ algorithm. $E_{U}$ represents spectrum efficiency of user $U$ and it is the reflection of channel quality; it can be obtained from users' Channel Quality Indicator (CQI) as shown in Fig. 2. $H_{U}^{i}$ is the history throughput of user $U$ service $i$ which is able to bring better fairness to the system. We can get it by imitating the method in conventional PF, and the formula is as follows.

$$
H_{U}^{i}=(1-\beta) * H_{U}^{i}+\beta * T_{U}^{i}
$$

Where, $T_{U}^{i}$ is data volume received successfully by user $U$ service $i$ in the last TTI, $\beta$ is the factor to compute history throughput, generally it is 0.01 .

Several services maybe available at the same time for one user, that is, there are several downlink Dedicated Traffic Channels (DTCH). Priority between them is determined by MAC Logical Channel Prioritization (MLP). We introduce $w_{i}$ to prioritize DTCH.

When setting factor $w_{i}$, both Signaling Radio Bearer (SRB) and Data Radio Bearer (DRB) should be taken into account. MLP of SRB is set default in protocol, SRB0 and SRB1 has been set 1 while SRB2 3. Priority of DRB is left to be set according to the specific circumstance. We put forward a method on account of the partition of services in protocol.

Suppose there are $\mathrm{m}$ kinds of DTCH, and MLP value 1, 2, 3 are left to SRB, then the rest $4 \sim \mathrm{m}$ can be used by DRB. For the DRB, a DTCH corresponds to a service while a service corresponds to a QCI value provided in protocol. Consequently, we set $\mathrm{MLP}=\mathrm{QCI}+3$. When $\mathrm{MLP}=i$, weight factor of logical channel priority can be calculated by

$$
w_{i}=n^{m-i}
$$

Where $n$ is a positive integer greater than 1 .

To reduce the delay and decrease the packet loss rate, we introduce a delay related factor $D_{U}^{i}$, which represents wait time of packet. The larger its value, the higher priority it corresponds to. This factor is capable of enhancing the scheduling priority of users who have longer wait time.

\section{B. Resource Allocation of Non-GBR Services}

We begin to allocate resource for Non-GBR services after all GBR services have been served or GBR available resource is exhausted. In view of their lower demand for QoS, and to reduce the complexity, we refer to the conventional PF algorithm as well as leave out delay and loss rate factors. Similarly, we add $w_{i}$ into our calculation to enable QCI high service get higher priority.

Priority calculation formula is as follows:

$$
F_{U}^{i}=w_{i} * \frac{E_{U}}{H_{U}^{i}}
$$

Where, $w_{i}$ is the weight factor of logical channel $i, E_{U}$ is spectrum efficiency of user $U, H_{U}^{i}$ is the history throughput of service $i$. These factors can be obtained in the same way as former.

What to note here is that, to avoid starvation, while still serving the logical channels according to their priorities, in LTE a Prioritized Bit Rate (PBR) is configured by the eNode $\mathrm{B}$ for each logical channel. That is to say, when allocating resource for users, RBs needed by PBR will be allocated in the first place. Only when all logical channels have been served up to their PBR, then if there is still room left in the MAC PDU each logical channel is served again in decreasing order of priority. In the process of secondary distribution, only logical channels that still have data can be served in decreasing order of priority. Priority mentioned here is the one we calculated before.

\section{Simulation Results And Performance Analysis}

\section{A. Simulation Parameters}

In this section, we evaluate performance of the proposed scheduling algorithm, called proposed PF. In the simulation, a total of 7 BS (cells) are considered in all simulations and users are randomly distributed in each cell. The available spectrum is $20 \mathrm{MHz}$ with carrier frequency $2.0 \mathrm{GHz}$. Related specifications are listed in Table I.

TABLE I. SyStEM SimULATION PARAMETERS

\begin{tabular}{ll}
\hline \multicolumn{1}{c}{ Parameter } & \multicolumn{1}{c}{ Setting/description } \\
\hline System Structure & $\begin{array}{l}\text { 7cells, 3sectors per } \\
\text { cell }\end{array}$ \\
\hline Distance between two sites & $500 \mathrm{~m}$ \\
\hline Radius of sector & $166.67 \mathrm{~m}$ \\
\hline BS height & $30 \mathrm{~m}$ \\
\hline Minimum distance from user to BS & $35 \mathrm{~m}$ \\
\hline Number of users per sector & $5 、 10 、 15 、 20 、 25$ \\
\hline
\end{tabular}




\begin{tabular}{lll}
\hline Frequency reuse number & 1 & \\
\hline System mode & TDD & \\
\hline Subframe configure & 1 & \\
\hline Carrier frequency & $2 \mathrm{GHz}$ & \\
\hline System bandwidth & $20 \mathrm{MHz}$ & \\
\hline Total RB number & 100 & \\
\hline Subcarrier number per RB & 12 & \\
\hline Transportation mode & $\begin{array}{l}\text { Open-loop } \\
\text { multiplexing }\end{array}$ & \\
\hline Antenna number & $2 * 2$ & \\
\hline Channel type & SCME & \\
\hline Shadow Fading Standard Deviation & $8 \mathrm{~dB}$ & \\
\hline
\end{tabular}

In order to show how the LTE MAC scheduler serves and handles the mixture of the different QoS services, users with three different QoS services (VoIP, Video and FTP) are defined, as shown in Table II. Some of them must be served with higher priority because of their real time nature. We investigate what happens if we mix all these services within one QoS class without distinguishing between the GBR and non-GBR, and what happens if we separate them. Meanwhile we set parameter $\alpha=0.5$.

TABLE II. SERVICES SETTING

\begin{tabular}{lll}
\hline \multicolumn{1}{c}{ Service name } & \multicolumn{1}{c}{ Service type } & QCI grade \\
\hline VoIP & GBR & 1 \\
\hline Video & GBR & 2 \\
\hline Ftp & Non-GBR & 8 \\
\hline
\end{tabular}

\section{B. Results Analysis}

Performance evaluation of scheduling algorithm contains three aspects: sector throughput, service delay and fairness between users. We get sector throughput by means of calculating transmitted data in sector, and service delay by packet wait time. Fairness between users can be computed via the following formula:

$$
F=\frac{\left(\sum_{\mathrm{i}=1}^{\mathrm{N}} \mathrm{x}_{\mathrm{i}}\right)^{2}}{\mathrm{~N} \sum_{\mathrm{i}=1}^{\mathrm{N}} x_{i}^{2}}
$$

Where, $x_{i}$ is the amount of data that service $i$ transmits successfully, $\mathrm{N}$ is the total number of service $i$. Suppose $F=1$, it means that the resource allocation can meet all of the users, and the system fairness is best. The higher the value of $F$ is, the better the fairness between users. The value of $F$ ranges from 0 to 1 .

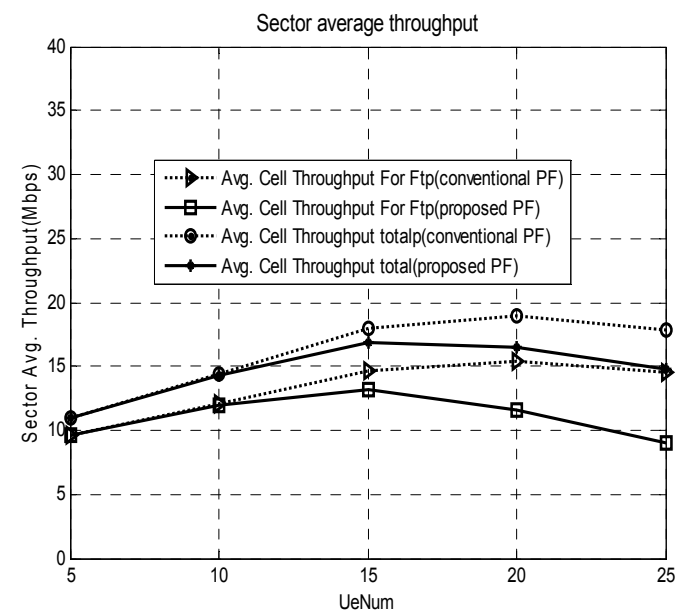

Figure 4. Throughput of sector and ftp

Fig. 4 shows that throughput of the sector and $\mathrm{ftp}$ in our proposed algorithm is lower than the conventional PF with the increase of user number. While there are few users in the system, all users' emergency telecommunication services can be served; there is no difference in throughput. However, with the user number in system increased, wireless resource is insufficient, in this case, the prioritization of VoIP and Video result in the decrease of ftp throughput. On the other hand, some of the RBs are allocated to users who have poor channel quality to transmit GBR data instead of serving users with high channel quality; this also leads to the reduction of the sector throughput.

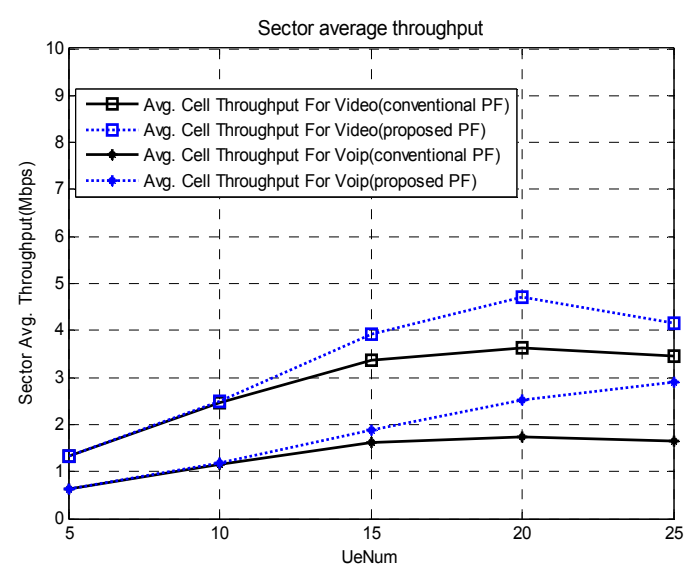

Figure 5. Throughput of VoIP/Video

In Fig. 5, throughput of VoIP and Video in the proposed algorithm is higher than the conventional evidently with the increase of users. This is because we allocate RBs for GBR data prior. This indicates that our algorithm is capable of improving throughput of GBR bearers. 


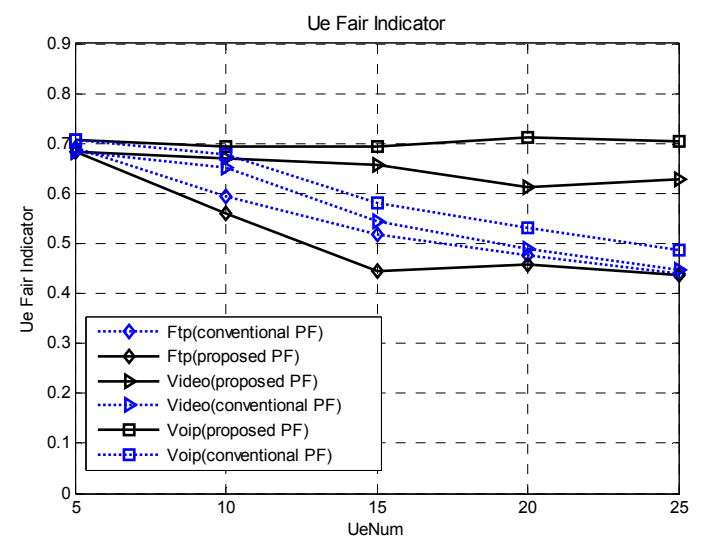

Figure 6. Comparison of service fairness

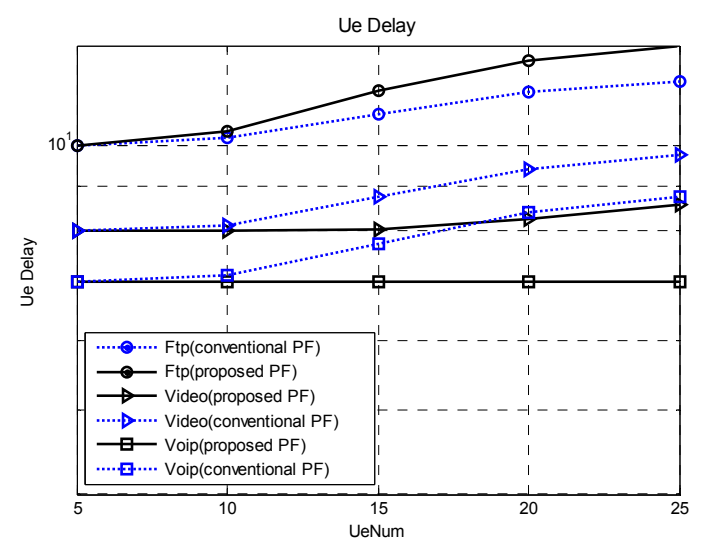

Figure 7. Comparison of service delay

Finally, service fairness and delay are shown in Fig. 6 and Fig. 7. Along with the number increase of users, at the cost of a certain amount of performance drawback of $\mathrm{ftp}$, Video and VoIP are capable of keeping preferable fairness and lower delay compared with conventional PF algorithm when applying the proposed. The key is that GBR service is prior to Non-GBR. This attains our goal that assures the QoS of real-time services at the cost of a certain amount of nonreal time services' performance.

\section{CONCLUSION}

In this paper, a novel resource scheduling algorithm based on difference of service QoS for LTE downlink has been proposed. The proposed MAC scheduler considers two main types of QoS bearers: Guaranteed Bit Rate (GBR) and Non Guaranteed Bit Rate (non-GBR). We assign RBs to GBR bearers ahead of Non-GBR to satisfy QoS of real-time service. We design a parameter to prevent GBR services from occupying too much resource when there are too many users in the system. A MAC logical channel prioritization weight factor is introduced to optimize the performance of service that has higher priority. The algorithm suits to not only real-time service but also the non-real-time, and simulation results show that it is able to improve the performance of GBR services at the cost of a certain amount of the non-real times'.

\section{ACKNOWLEDGEMENT}

This paper is supported by NSFC ( 61201134 , 61201135 ) , the Fundamental Research Funds for the Central Universities (72124669) and the 111 Project(B08038)

\section{REFERENCES}

[1] Z. Shen, J. G. Andrews, and B. L. Evans. "Adaptive resource allocation in multiuser OFDM systems with proportional fairness", IEEE Trans. On Wireless Comm., vol. 4, no. 6. pp. 2726-2737, Nov. 2005.

[2] Daiqin Yang, Dongxu Shen, Wenjian Shao and Li, V.O.K, "Towards opportunistic fair scheduling in wireless networks," IEEE ICC'06, vol. 11, pp. 5217-5221, Jun. 2006.

[3] Proebster, Magnus, Mueller, Christian M., Bakker, Hajo, “Adaptive fairness control for a proportional fair LTE scheduler," IEEE PIMRC, pp. 1504-1509, 2010.

[4] Ye, Fang; Zhang, Huan; Gu, Yunbiao, “An improved proportional fair resource scheduling algorithm for LTE system," Journal of Computational Information Systems, vol. 8, no. 3, pp. 1317-1323, March 2012

[5] F. M. Chiussi and V. Sivaraman, "Achieving high utilization in guaranteed services networks using Early-Deadline-First scheduling," 6th Int'l. Wksp. QoS, pp. 18-20, May 1998.

[6] Ameigeiras, Pablo; Wigard, Jeroen; Mogensen, Proben, "Performance of the M-LWDF scheduling algorithm for streaming services in HSDPA," IEEE Vehicular Technology Conference, vol. 60, no. 2, pp. 999-1003, 2004.

[7] Xian, Yong-Ju; Tian, Feng-Chun; Xu, Chang-Biao; Yang, Yue; "Analysis of M-LWDF fairness and an enhanced M-LWDF packet scheduling mechanism," Journal of China Universities of Posts and Telecommunications, vol. 18, no. 4, pp. 82-88, August 2011.

[8] Ju Yong Lee, Sorour, S.; Valaee, S.; "Dynamic parameter adaptation for M-LWDF/M-LWWF scheduling," IEEE Transactions on Wireless Communications, vol. 11, no. 3, pp. 927-937, March 2012.

[9] 3GPP TS36.211. V8.0.0, "Evolved Universal Terrestrial Radio Access (E-UTRA); Physical Channels and Modulation," www.3gpp.org. Oct, 2010.

[10] 3GPP TS36.300. v10.1.0. "Evolved Universal Terrestrial Radio Access (E-UTRA) and Evolved Universal Terrestrial Radio Access Network (E-UTRAN)," www.3gpp.org, Oct, 2010.

[11] 3GPP TS36.321. v8.5.0. "Evolved Universal Terrestrial Radio Access (E-UTRA) Medium Access Control (MAC) Protocol specification (Release 8) [S],” www.3gpp.org, 2009-03. 